\title{
Fundamental Insights into Graphene Strain Sensing
}

\section{(Supporting Information)}

\author{
Mufeng Liu ${ }^{1, \sharp}$, Zheling Li $^{1, \text {,*, }}$, Xin Zhao ${ }^{1,2}$, Robert J. Young ${ }^{1, *}$, Ian A. Kinloch $^{1}$ \\ 1. National Graphene Institute/Department of Materials, University of Manchester, \\ Manchester M13 9PL, UK
}

2. BTR New Material Group Co., Ltd., BTR Industrial Park, Xitian, Gongming, Guangming District, Shenzhen, China

Corresponding authors:

Zheling Li (zheling.li@manchester.ac.uk)

Robert J. Young (robert.young@manchester.ac.uk)

\section{S1. Experimental Method}

The graphene flakes were obtained by exfoliating graphite crystals (NGS Naturgraphit GmbH) using the 'Scotch tape' method. ${ }^{1}$ The exfoliated graphene flakes were then deposited onto PMMA beams (Solaris S000, Panelgraphic) that were cleaned by $\mathrm{H}_{2} \mathrm{O}$ followed by UV-Ozone treatment. The specimen shown in Figure 5 in the main text, after the graphene was deposited onto PMMA target, has been subjected to an annealing process at $\sim 110^{\circ} \mathrm{C}$, followed by cooling down to room temperature. The specimen was then placed in a four-point bending rig designed 
in-house, and the strain applied step-wise. The slow growth of crazes $^{2}$ was carefully monitored. Once they grew to reach graphene, the deformation process was paused and the mapping using Raman spectroscopy was undertaken.

The in-situ bending experiment was carried out on the microscope stage of a Raman spectrometer (laser $\lambda=488 \mathrm{~nm}$, Horiba LabRAM HR Evolution) where the laser was illuminating perpendicular to the specimen. ${ }^{3}$ The mapping experiment was carried out using an automated stage with a step size between 0.6 0.8 $\mu \mathrm{m}$. Optical images of the graphene flakes were obtained using the Labspec software on the Raman spectrometer with a filter in place to enhance the contrast.

During bending, crazes initiate from existing defects when the localized strain succeeds the craze strain of the polymer, which is a material property of the PMMA. ${ }^{2}$ Considering the similar strain applied to the area covered by graphene and its vicinity in the four-point bending set-up, it is possible for crazing to occur at different locations, partially determined by the location of possible existing defects in the polymer.

\section{S2. Raman Spectroscopy}

Raman spectroscopy can be used as a technique to determine the strain in graphene with high sensitivity. It can be evaluated in terms of the 'spectral resolution' and the 'spatial resolution' (lateral not depth, hereafter), hence good sensitivity means the sensor is sensitive to 'small' strain variation, occurring in a 'small' area. The 'spectral resolution' of Raman spectrometer is $\sim 1 \mathrm{~cm}^{-1}$, corresponding to a strain variation of $\sim 0.016 \%$ as calculated from the calibration between the Raman 2D band position and strain as discussed in the main text based on the knowledge of Grüneisen parameter. ${ }^{4}$ The 'spatial resolution' $A=0.61 \lambda / \mathrm{NA}$, where $\lambda(=488 \mathrm{~nm})$ is the wavelength of the laser, and NA is the numerical aperture of the objective. An objective 
with $\mathrm{NA}=0.9$ leads to $A \approx 330 \mathrm{~nm}$. However, this is just the diffraction limit and the practical value can be about 2-3 times higher. ${ }^{5}$ The Raman spectra of the monolayer and bilayer graphene are shown in Figure S1. ${ }^{6}$

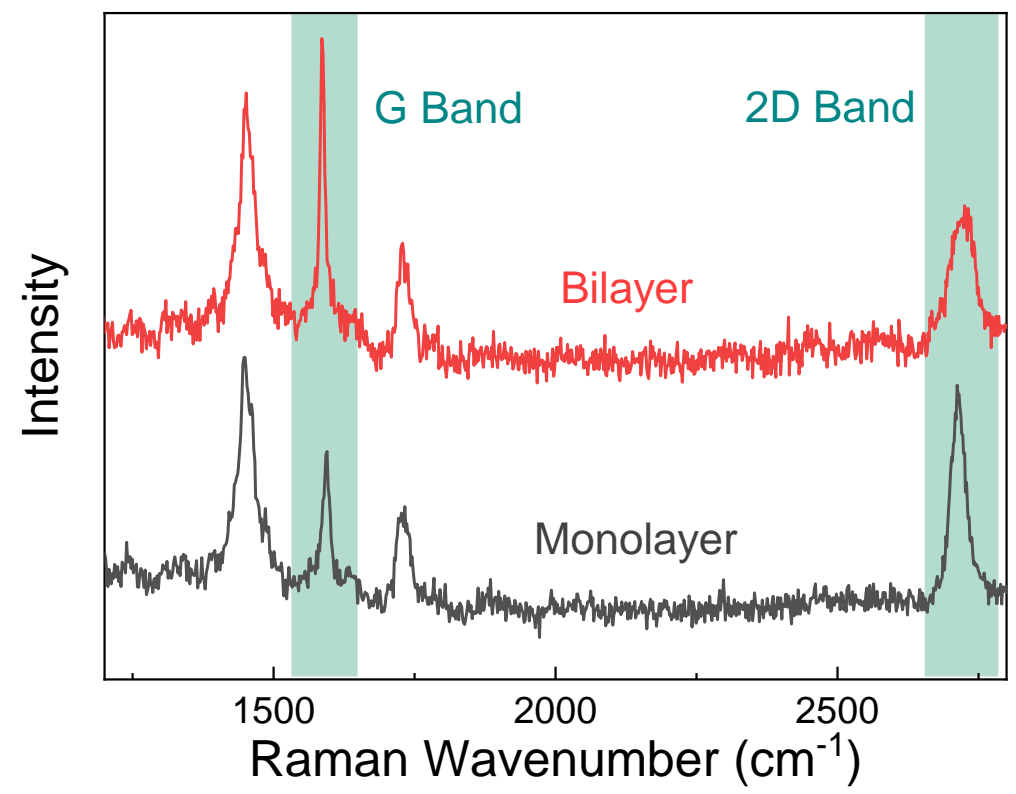

Figure S1. Representative Raman spectra of monolayer and bilayer graphene.

S3. Deformation Test Monitored In-situ by Raman Spectroscopy and Shear-Lag Analysis

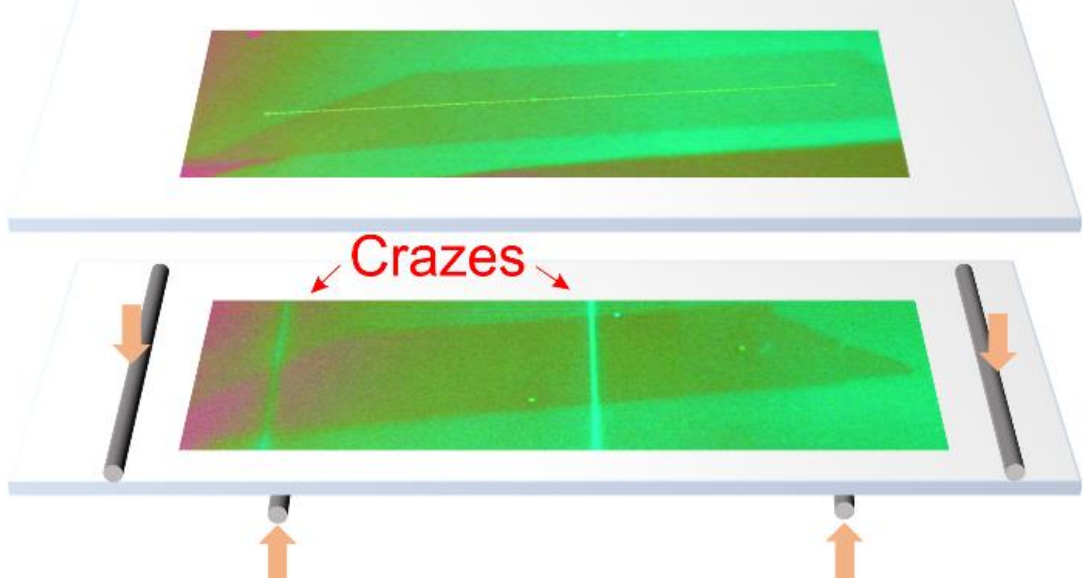

Figure S2. Schematic diagram of a graphene flake being deformed on the surface of a PMMA beam (subject to a four-point bending process), where strain-induced crazes initiated across the flake, perpendicular to the strain direction. 
Both the Raman 2D and G band bands downshift significantly as strain increased along with the splitting of $\mathrm{G}$ band at large strain (Figure S3). ${ }^{4}$

(a)

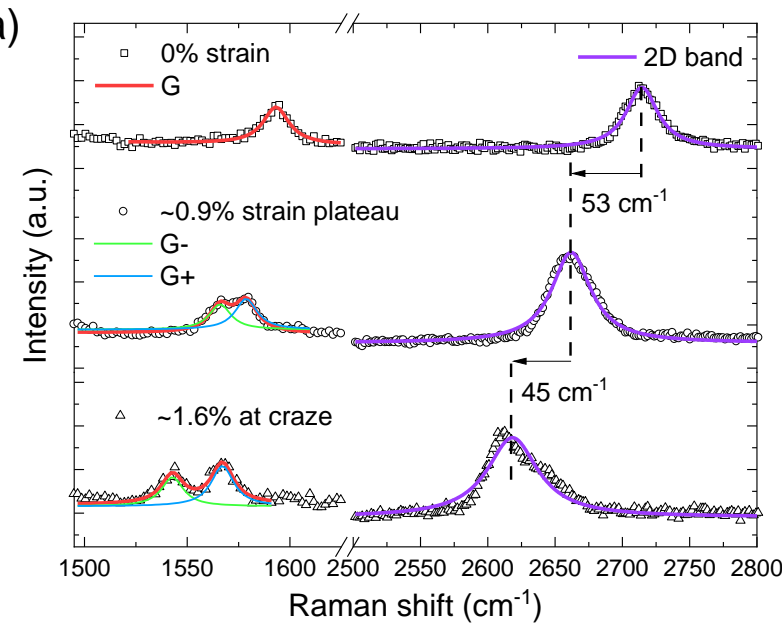

(b)

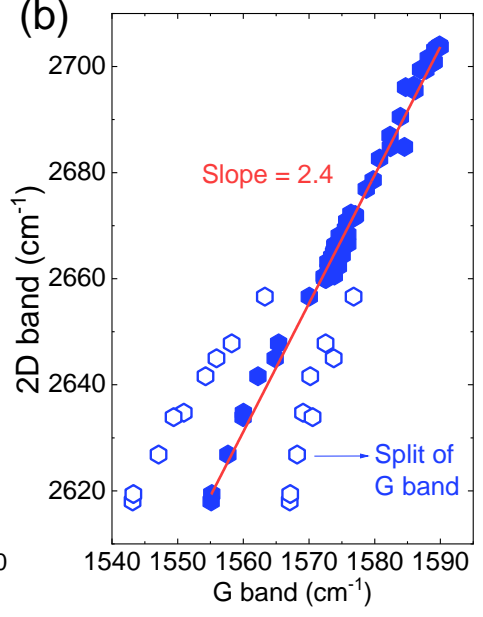

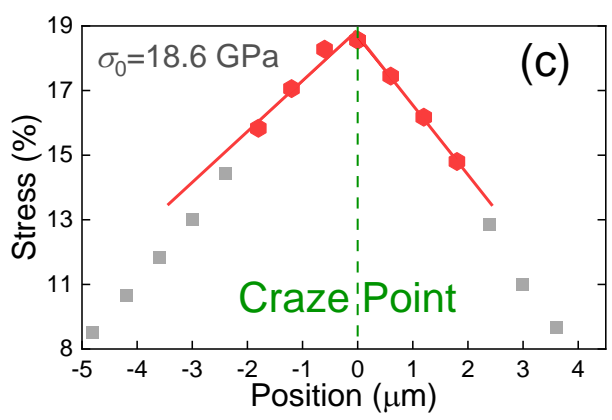

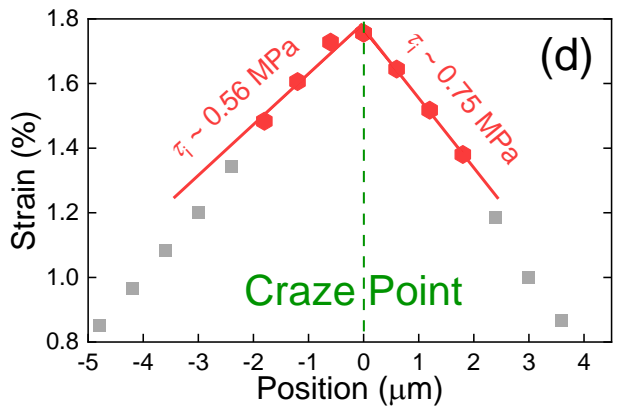

Figure S3. (a) Representative Raman spectra of graphene at $0 \%, 0.9 \%$ strain plateau and $1.6 \%$ strain at craze point. (b) Correlation of the position of Raman 2D band and G band. The blue points also denote the average of G- and $\mathrm{G}+$ band positions if split of $\mathrm{G}$ band occurs (open blue dots). (c) Stress and (d) Strain distribution of the monolayer graphene in Figure 1a obtained from Raman 2D band shift against the position along the flake $x$, at an applied strain of $0.9 \%$. In (c) the $\sigma_{0}$ was determined to be $\sim 18.6 \mathrm{GPa}$. In (d) the interfacial shear stress was determined to be $0.56 \mathrm{MPa}$ and $0.75 \mathrm{MPa}$ by linear fittings of both sides using Equation 1, respectively.

As has been well-established both experimentally and theoretically previously by considering the effect of strain on the vibrational modes based on the knowledge of Grüneisen parameter, the downshift of Raman 2D band position is proportional to the strain applied to the graphene, i.e. the Raman 2D band of monolayer graphene downshifts $\sim 60 \mathrm{~cm}^{-1} / \%$ strain under uniaxial tension. ${ }^{4,7}$ Based on this, the Raman 2D band position of the graphene flakes can be converted 
into the strain. For graphene with an intact interface, the strain distribution can be fitted using shear-lag theory:

$$
\varepsilon_{\mathrm{g}}=\varepsilon_{\mathrm{m}}\left[1-\frac{\cosh \left(n s_{\frac{x}{l}}\right)}{\cosh \left(\frac{n s}{2}\right)}\right] \quad \text { where } n=\sqrt{\frac{2 G_{\mathrm{m}}}{E_{\mathrm{g}}}\left(\frac{t}{T_{\mathrm{RVE}}}\right)}
$$

where $l$ and $s(=l / t)$ are the length and aspect ratio of the flake of the bonded region, respectively; $\varepsilon_{\mathrm{m}}$ is the strain in the monolayer graphene with position $x$ and $G_{\mathrm{m}}$ is the shear modulus of the substrate. The shear lag fittings show excellent consistency with the experimental data at an applied strain of around $0.9 \%$ (Figure 1b). The plateau of strain distribution of graphene equals to the applied strain in the polymer, therefore as mentioned in the main text, the strain at the PMMA surface is also $\sim 0.9 \%$. Details of the shear-lag analysis can be found elsewhere. ${ }^{8}$

The strain measured by this method relates to the intrinsic properties of graphene, and it does not rely on the results from other measurements where uncertainties can potentially occur. For example, the strain measured from the separation of the grips may vary with the adhesion between the graphene and grips and even with complex local stress/strain, it may therefore not be necessarily the same as that applied to the graphene.

In fact in many studies, when strain was applied using other methods, the 'real' strain in graphene has commonly been calibrated and benchmarked by using Raman spectroscopy as demonstrated in this work. ${ }^{9-12}$ This further demonstrates the accuracy of using theoretical value as a comparison to predict strain as shown in this work, which is of vital importance for revealing the fundamentals of strain sensing, where other side effects can be ruled out. This is also crucial to evaluate the strain sensors for applications in devices where local strain variation occurs. 


\section{S4. Craze Analysis}

If $\delta$ is the crack opening displacement (COD) at the tip of craze or crack, ${ }^{2}$ since the graphene is fully attached to the PMMA substrate outside the debonding region, then the mean strain of the graphene is given by

$$
\begin{gathered}
\overline{\varepsilon_{\text {debond }}}=\frac{\varepsilon_{\text {debond }}}{2}=\frac{\delta}{2 a} \\
\frac{\varepsilon_{\text {debond }}}{\mathrm{d} x}=\frac{\tau_{\mathrm{i}}}{t E_{\mathrm{g}}}=\frac{\varepsilon_{\text {debond }}}{a}=K
\end{gathered}
$$

The parameter $K$ is a material constant that only relates to $\tau_{\mathrm{i}}, E_{\mathrm{g}}$ and $t$. Combining Eq.(S2) and (S3) gives:

$$
\varepsilon_{\text {debond }}=\sqrt{\delta \cdot K}
$$

Combining Eq.(S2) and (S4) leads to:

$$
a=\sqrt{\frac{\delta}{K}}
$$

Therefore, the strain distribution measured by using Raman spectroscopy can be used to determine the value of the constant $K . K$ together with the $\varepsilon_{\text {debond }}$ also measured by Raman spectroscopy enable the estimation of the $\operatorname{COD}(\delta(r))$, even when the $\operatorname{COD}(\delta(r))$ is smaller than the spatial resolution of the Raman spectrometer.

\section{S5. Energy Criterion}

The energy criterion model is shown in Figure S4. It is modified from the theory of fiber bridging of crack during pull-out, developed earlier. ${ }^{13,}{ }^{14}$ The monolayer graphene on the surface of the substrate has a width of $w$ and thickness of $t$ (Figure S4a and b). The monolayer graphene along with the substrate has an overall thickness of $T$ (Figure S4c). A craze (or crack) 
in the substrate crosses the flake at the interface in a direction perpendicular to the loading axis.

Figure S4d shows that the debonded length is defined as $a$ and the bridging stress as $\sigma_{0}$.

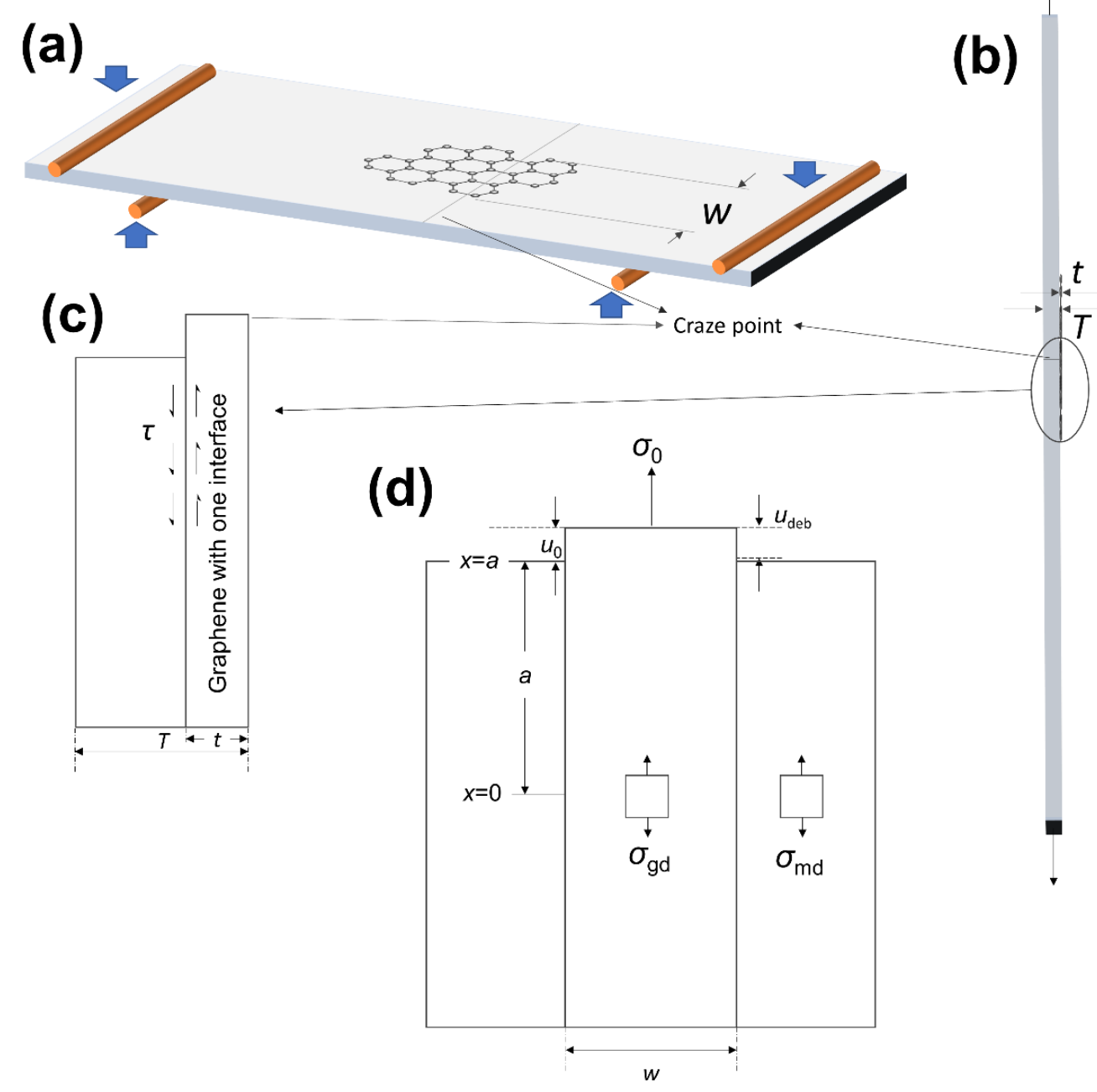

Figure S4. (a-b) Schematic diagrams of a monolayer graphene on the polymer surface where a craze lies across the flake in the width direction, perpendicular to the axial direction. This case is defined as craze/crack bridging of monolayer graphene in the nanocomposite; (c-d) A representative volume element for energy-based criterion showing a loaded graphene flake bridging a craze.

For the bonded interface, based on the rule-of-mixtures, the equilibrium axial stresses in the flake and the matrix, $\sigma_{\mathrm{g}}$ and $\sigma_{\mathrm{m}}$ are given by

$$
\sigma_{\mathrm{g}}=\frac{V_{\mathrm{g}} E_{\mathrm{g}} \sigma_{0}}{E_{\mathrm{c}}}
$$




$$
\sigma_{\mathrm{m}}=\frac{V_{\mathrm{f}} E_{\mathrm{m}} \sigma_{0}}{E_{\mathrm{c}}}
$$

where $E_{\mathrm{g}}$ and $E_{\mathrm{m}}$ are modulus of the graphene and the matrix, respectively; $E_{\mathrm{c}}$ is the modulus of the composite; $V_{\mathrm{g}}$ and $V_{\mathrm{m}}$ are the volume fraction of the graphene and the matrix, respectively. At the end of the debonded region, the axial stresses, $\sigma_{\mathrm{gd}}$ and $\sigma_{\mathrm{md}}$ are obtained from stress transfer theory as

$$
\begin{gathered}
\sigma_{\mathrm{gd}}=\sigma_{0}-\frac{a \tau_{\mathrm{i}}}{t} \\
\sigma_{\mathrm{md}}=\frac{a V_{\mathrm{g}} \tau_{\mathrm{i}}}{t V_{\mathrm{m}}}
\end{gathered}
$$

Assuming $\tau_{\mathrm{i}}$ is constant, the axial stresses of the graphene and the matrix along the debonded length are given by

$$
\begin{gathered}
\sigma_{\mathrm{ga}}=\sigma_{\mathrm{gd}}+\frac{x\left(\sigma_{0}-\sigma_{\mathrm{gd}}\right)}{a} \\
\sigma_{\mathrm{ma}}=\left(1-\frac{x}{a}\right) \sigma_{\mathrm{md}}
\end{gathered}
$$

The debonding criterion can be obtained using the energy balance condition:

$$
\mathrm{d} W=\mathrm{d} U_{\mathrm{e}}+\mathrm{d} U_{\mathrm{s}}+\mathrm{d} G_{\mathrm{i}}
$$

where $W$ is the work done by the interfacial debonding with a bridging stress of $\sigma_{0}, U_{\mathrm{e}}$ is the elastic energy for the graphene in the composite; $U_{\mathrm{s}}$ represents the sliding energy dissipated, resulting from graphene/matrix displacement at constant interfacial shear stress $\tau_{\mathrm{i}}$, and finally $G_{\mathrm{i}}$ refers to the interfacial energy.

The elastic energy change for the graphene in the composite contains two components, $\mathrm{d} U_{\mathrm{eb}}$ and $\mathrm{d} U_{\text {ed }}$ for the bonded and debonded sections, respectively. For the bonded section, the elastic energy can be calculated from the elastic strain density. When the debonded length advances a 
distance of $\mathrm{d} a$, the bonded region is also reduced by a length of $\mathrm{d} a$ and a volume of $T w \mathrm{~d} a$ and therefore,

$$
\mathrm{d} U_{\mathrm{eb}}=-\frac{T w}{2}\left(\frac{V_{\mathrm{g}} \sigma_{\mathrm{g}}^{2}}{E_{\mathrm{g}}}+\frac{V_{\mathrm{m}} \sigma_{\mathrm{m}}^{2}}{E_{\mathrm{m}}}\right) \mathrm{d} a
$$

Substituting Equation (S6) and (S7) into (S13),

$$
\mathrm{d} U_{\mathrm{eb}}=-\frac{t w V_{\mathrm{g}} \sigma_{0}^{2}}{2 E_{\mathrm{c}}} \mathrm{d} a
$$

In the debonded region, the elastic strain density of the graphene and the matrix is given by

$$
U_{\text {ed }}=T w \int_{0}^{a}\left(\frac{V_{\mathrm{g}} \sigma_{\mathrm{ga}}^{2}}{E_{\mathrm{g}}}+\frac{V_{\mathrm{m}} \sigma_{\mathrm{ma}}^{2}}{E_{\mathrm{m}}}\right) \mathrm{d} x
$$

Substituting equations (S5) and (S6) into (S15) and integrating (S15) and differentiating with respect to $a$ :

$$
\mathrm{d} U_{\text {ed }}=\frac{t w}{2 E_{\mathrm{g}}}\left(\sigma_{0}^{2}-\frac{2 a \tau \sigma_{0}}{t}+\frac{a^{2} \tau_{\mathrm{i}}^{2} E_{\mathrm{c}}}{t^{2} V_{\mathrm{m}} E_{\mathrm{m}}}\right) \mathrm{d} a
$$

and therefore

$$
\mathrm{d} U_{\mathrm{e}}=\mathrm{d} U_{\mathrm{eb}}+\mathrm{d} U_{\text {ed }}=\frac{t w V_{\mathrm{m}} E_{\mathrm{m}}}{2 E_{\mathrm{g}} E_{\mathrm{c}}}\left(\sigma_{0}-\frac{a \tau_{\mathrm{i}} E_{\mathrm{c}}}{\mathrm{t} V_{\mathrm{m}} E_{\mathrm{m}}}\right)^{2} \mathrm{~d} a
$$

The relative displacement between the graphene and the matrix leads to the sliding energy, $U_{\mathrm{s}}$, dissipated at constant shear stress $\tau_{\mathrm{i}}$. Hence,

$$
U_{\mathrm{s}}=w \int_{0}^{a} \tau_{\mathrm{i}}\left(w_{\mathrm{g}}-w_{\mathrm{m}}\right) \mathrm{d} x
$$

where $w_{\mathrm{f}}$ and $w_{\mathrm{m}}$ are the respective axial displacements in the graphene and the matrix due to the axial stresses in equation (S10) and (S11), which can be obtained from integration of the function for axial deformation,

$$
w_{\mathrm{g}}=\frac{x \sigma_{\mathrm{gd}}}{E_{\mathrm{g}}}+\frac{x^{2}\left(\sigma_{0}-\sigma_{\mathrm{gd}}\right)}{2 a E_{\mathrm{g}}}
$$




$$
w_{\mathrm{m}}=\left(x-\frac{x^{2}}{2 a}\right) \frac{\sigma_{\mathrm{md}}}{E_{\mathrm{m}}}
$$

Substituting Equations (S19) and (S20), integrating and differentiating $U_{\mathrm{s}}$ with respect to $a$ gives

$$
\mathrm{d} U_{\mathrm{s}}=w \tau_{\mathrm{i}}\left(\frac{a \sigma_{0}}{E_{\mathrm{g}}}-\frac{a^{2} \tau_{\mathrm{i}} E_{\mathrm{c}}}{t V_{\mathrm{m}} E_{\mathrm{m}} E_{\mathrm{g}}}\right) \mathrm{d} a
$$

The work done, $W$, due to the interfacial debonding with a bridging stress, $\sigma_{0}$, is,

$$
W=t w \sigma_{0} u_{\mathrm{deb}}
$$

where $u_{\text {deb }}$ is given by

$$
u_{\mathrm{deb}}=\frac{a V_{\mathrm{m}} E_{\mathrm{m}} \sigma_{0}}{E_{\mathrm{g}} E_{\mathrm{c}}}-\frac{a^{2} \tau_{\mathrm{i}}}{2 t E_{\mathrm{g}}}
$$

Substituting Equation (S22) into (S23) and differentiating with respect to $a$, gives

$$
\mathrm{d} W=t w \sigma_{0}\left(\frac{V_{\mathrm{m}} E_{\mathrm{m}} \sigma_{0}}{E_{\mathrm{g}} E_{\mathrm{c}}}-\frac{a \tau_{\mathrm{i}}}{t E_{\mathrm{g}}}\right) \mathrm{d} a
$$

The change in the interfacial energy is

$$
\mathrm{d} G_{\mathrm{i}}=w G_{\mathrm{i}} \mathrm{d} a
$$

since the debonded length advances $\mathrm{d} a$ and therefore the debonded interface area is $w \mathrm{~d} a$. Based on the energy balance condition defined in Equation (S12), combining Equations (S21), (S24) and (S25), the bridging stress, $\sigma_{0}$, is given by

$$
\sigma_{0}=\left(\frac{2 G_{\mathrm{i}} E_{\mathrm{g}} E_{\mathrm{c}}}{t V_{\mathrm{m}} E_{\mathrm{m}}}\right)^{\frac{1}{2}}+\frac{a \tau_{i} E_{\mathrm{c}}}{t V_{\mathrm{m}} E_{\mathrm{m}}}
$$

For monolayer/bi-layer graphene on a substrate, it is reasonable to assume that: $E_{\mathrm{c}} \approx E_{\mathrm{m}}$ and $V_{\mathrm{m}} \approx 1$. Hence, Equation $\quad(\mathrm{S} 26)$ can be simplified and transformed into, 


$$
\sigma_{0}=\left(\frac{2 G_{\mathrm{i}} E_{\mathrm{g}}}{t}\right)^{1 / 2}+\frac{a \tau_{\mathrm{i}}}{t}
$$

where a linear relationship between the bridging stress $\left(\sigma_{0}\right)$ and the product of debonded length and the interfacial shear stress of the debonded area $\left(a \tau_{\mathrm{i}}\right)$, is predicted. The interfacial energy $\left(G_{\mathrm{i}}\right)$ can be determined by a simple calculation because the modulus $\left(E_{\mathrm{g}}\right)$ and the thickness $(t)$ of the monolayer graphene are known. Accordingly, the maximum strain at the crazing location $\varepsilon_{0}$ is given as:

$$
\varepsilon_{0}=\left(\frac{2 G_{\mathrm{i}}}{t E_{\mathrm{g}}}\right)^{1 / 2}+\frac{a \tau_{\mathrm{i}}}{t E_{\mathrm{g}}}
$$

\section{S6. Application of the Modelling on Different Flakes}

Graphene with different numbers of layers responds differently to crazing of the polymer substrate. It can be seen in Figure S5a that a craze penetrated two ribbon-like monolayers at an applied strain of $\sim 0.85 \%$. The strain distribution showed a similar stress concentration in the craze plane but with a value $\sim 2.3 \%$ (Figure S5b), higher than the flake shown in Figure 1 in the main text. Again the correlation of the Raman 2D band and $\mathrm{G}$ positions indicates the absence of doping of graphene (Figure S5c). ${ }^{15}$ It is worth noting that the strain achieved, 2.3\%, is, to date, the largest uniaxial strain that has been reported for supported graphene. ${ }^{16}$ It demonstrates the potential of utilizing stress concentration for bandgap opening, which could lead the way to the future fabrication of strain engineered devices using $2 \mathrm{D}$ materials. ${ }^{17-19}$

Figure S5d shows a bilayer graphene flake, where the craze only penetrated partially into the flake region. Its strain distribution shows a high strain concentration $\sim 1.6 \%$ at the point where the craze crosses the flakes, at an applied strain of about 1.2\% (SCF 1.3). It diminishes radially from where the craze crosses the flake, similar to the behavior seen in Figure 1a in the main text. 

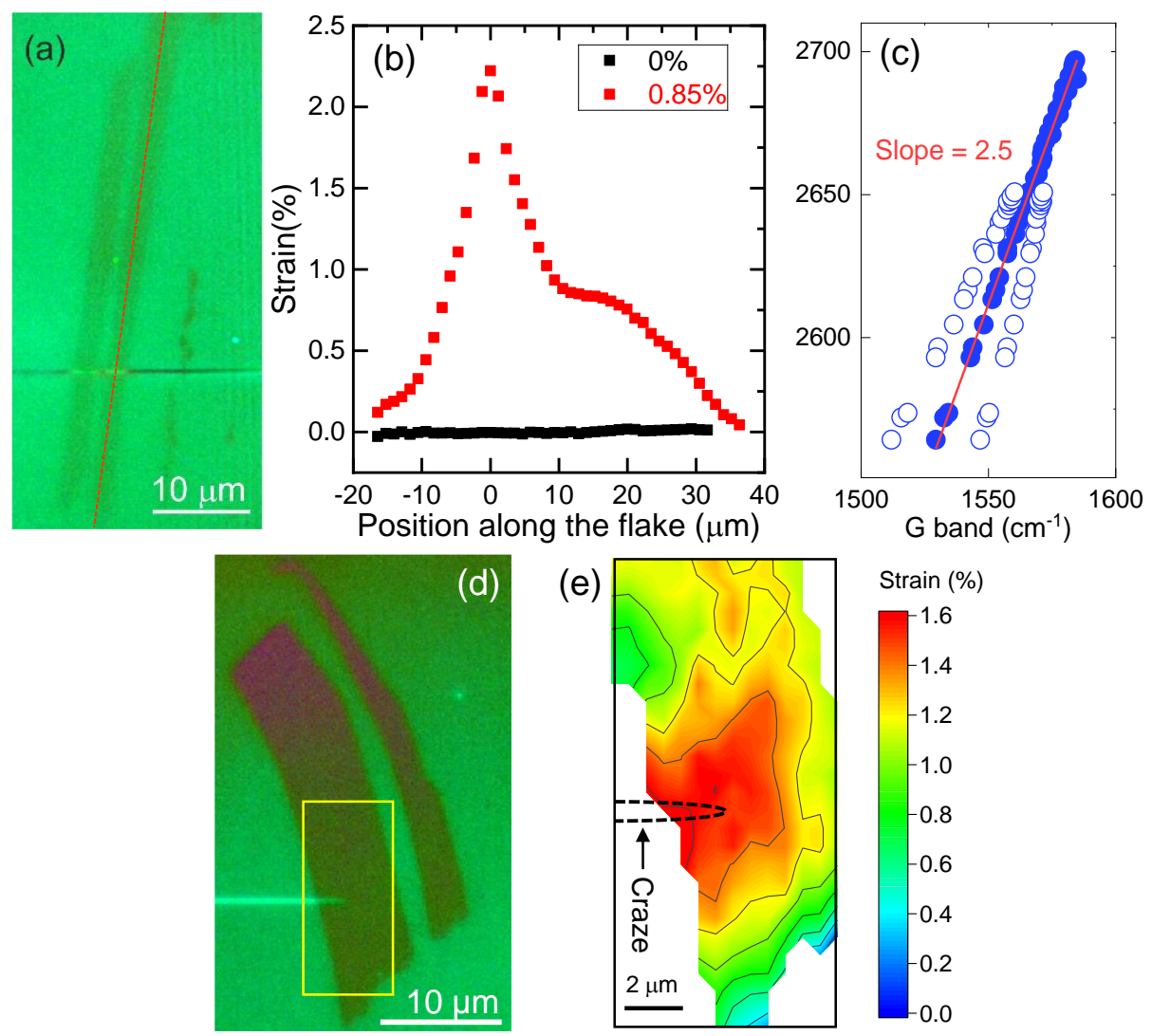

(e)

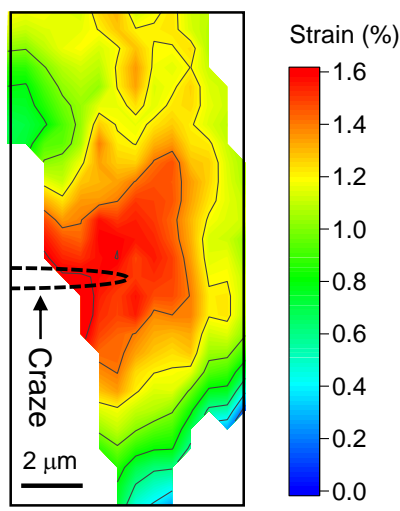

Figure S5 (a) False color optical micrograph of ribbon-like monolayers deformed at $0.85 \%$ with a craze throughout the flake. (b) Strain distribution along the red line shown in (a) at $0 \%$ and $0.85 \%$ applied strain. (c) Correlation of the $2 \mathrm{D}$ and $\mathrm{G}$ band position; (d) False color optical micrograph of a bi-layer graphene. (e) Areal strain mapping of the flake at $\sim 1.2 \%$ applied strain in the region highlighted by the yellow frame in (d).

Table S1. Results for all the flakes (monolayers and bi-layers) analyzed in this work, including bridging stress $\sigma_{0}$, interfacial shear stress at debonded region $\tau_{\text {i }}$, debonded length $a$, interfacial bond energy $G_{\mathrm{i}}$.

\begin{tabular}{lllllll}
\hline Flake & Layer & $\sigma_{0}(\mathrm{GPa})$ & $\varepsilon_{0}(\%)$ & $\tau_{\mathrm{i}}(\mathrm{MPa})$ & $a(\mu \mathrm{m})$ & $G_{\mathrm{i}}\left(\mathrm{J} / \mathrm{m}^{2}\right)$ \\
\hline 1 & Mono- & $18.6 \pm 0.1$ & $1.77 \pm 0.01$ & $0.56 / 0.75$ & 1.8 & $3.6 \times 10^{-2}$ \\
\hline 2 & Mono- & $23.8 \pm 0.3$ & $2.25 \pm 0.01$ & $0.91 / 0.70$ & 3.5 & $3.7 \times 10^{-2}$ \\
\hline 3 & Mono- & $21.6 \pm 0.5$ & $2.06 \pm 0.05$ & $0.70 / 0.49$ & 3.4 & $3.9 \times 10^{-2}$ \\
\hline 4 & Bi- & $13.5 \pm 0.3$ & $1.54 \pm 0.03$ & $0.30 / 0.62$ & 1.9 & $5.7 \times 10^{-2}$ \\
\hline 5 & Bi- & $13.4 \pm 0.1$ & $1.54 \pm 0.01$ & $0.67 / 0.88$ & 2.1 & $4.7 \times 10^{-2}$ \\
\hline 6 & Bi- & $12.9 \pm 0.1$ & $1.47 \pm 0.01$ & $0.73 / 0.54$ & 1.5 & $5.0 \times 10^{-2}$ \\
\hline
\end{tabular}


The results upon a number of mono- and bi-layer graphene flakes are given in Table S1 and their details shown in Figure S6 - Figure S10. In the calculation for bilayer graphene, an interlayer stress transfer efficiency $\sim 0.8^{20}$ leads to a Young's modulus of $\sim 875 \mathrm{GPa}^{20,21}$ and a Raman 2D band shift rate of $\sim 50 \mathrm{~cm}^{-1} / \%$ strain. $^{20,22}$
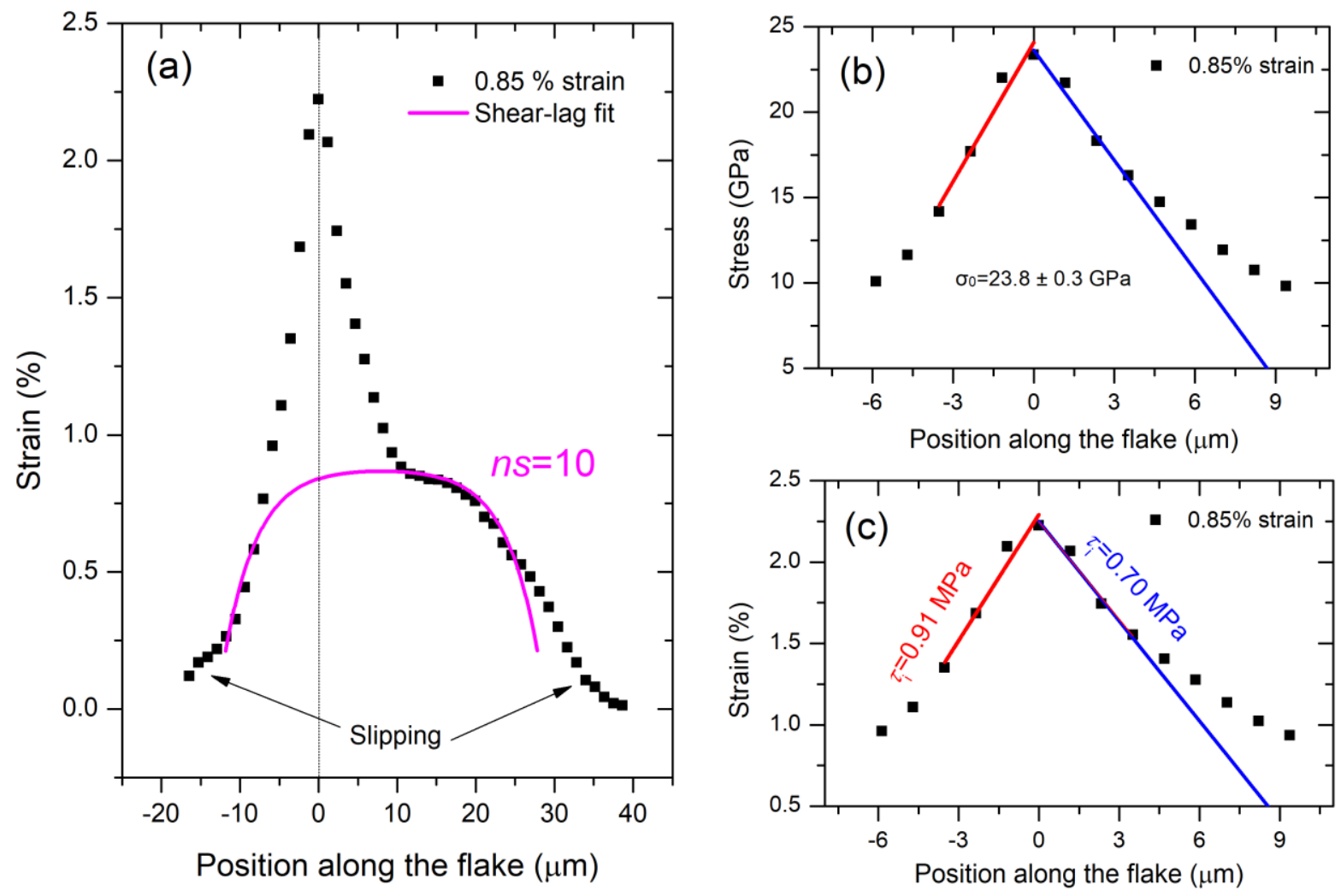

Figure S6. Analysis of flake No.2 (Table S1). 

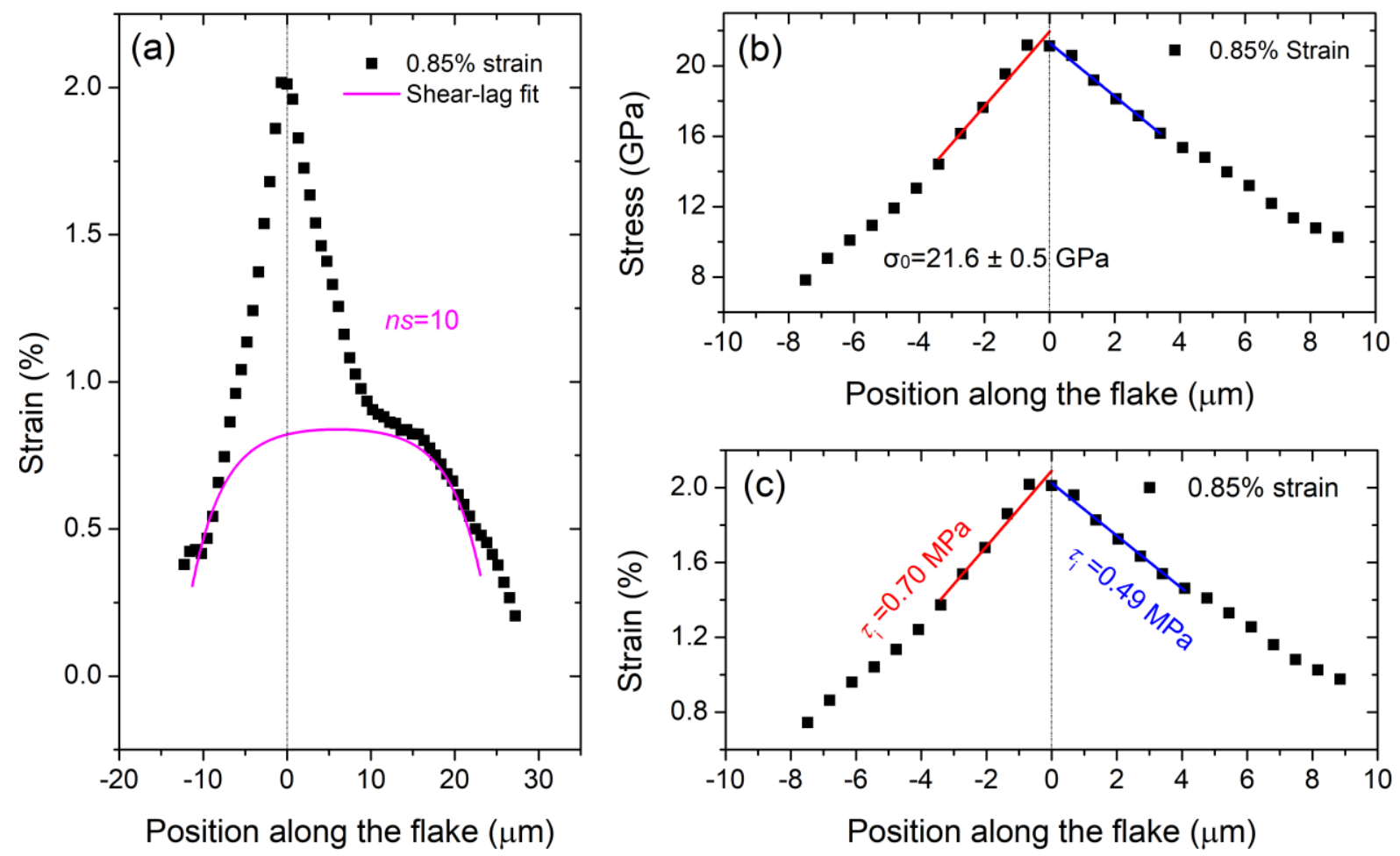

Figure S7. Analysis of flake No.3 (Table S1).

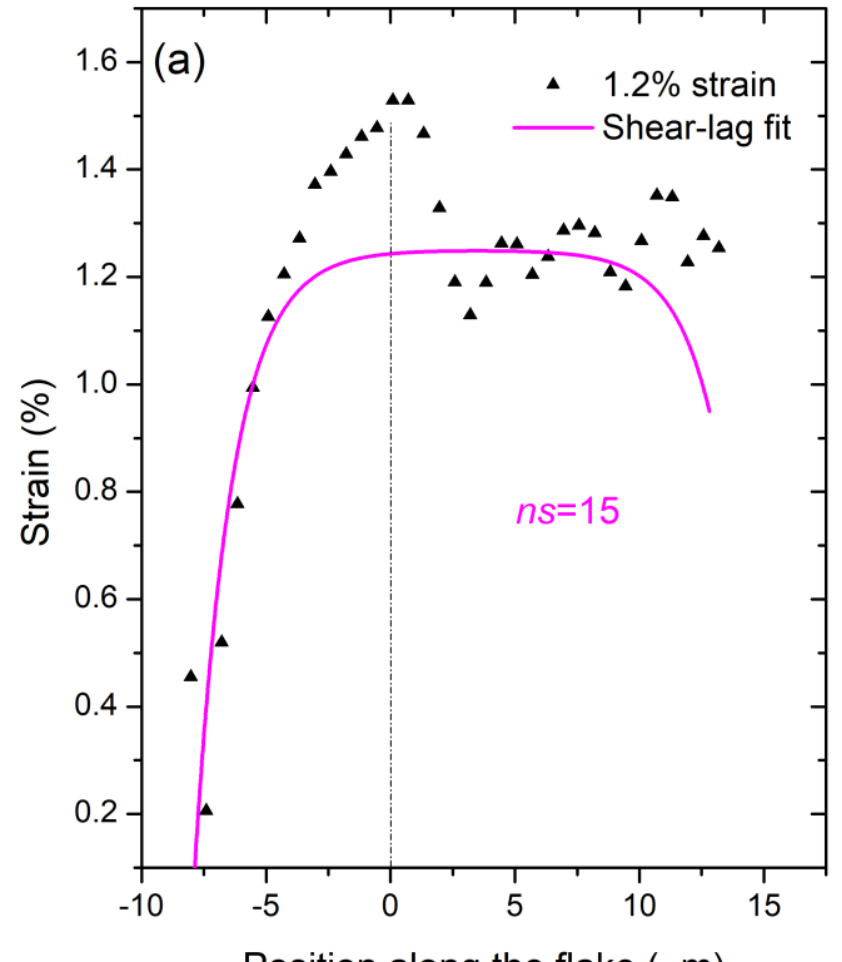

Position along the flake $(\mu \mathrm{m})$
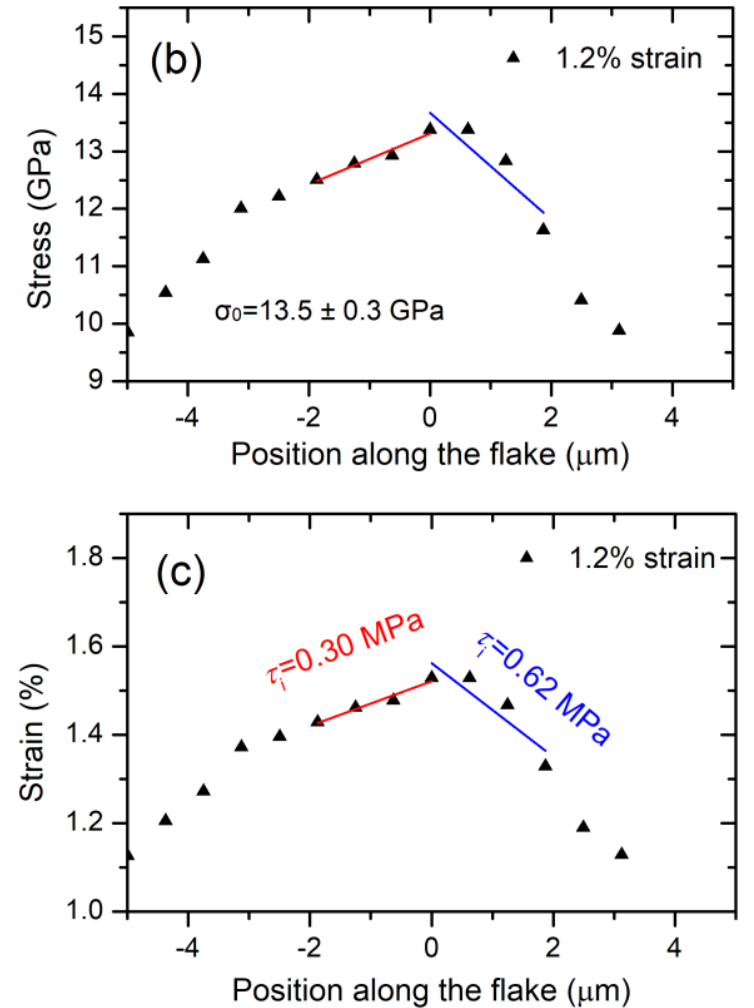

Figure S8. Analysis of flake No.4 (Table S1). 

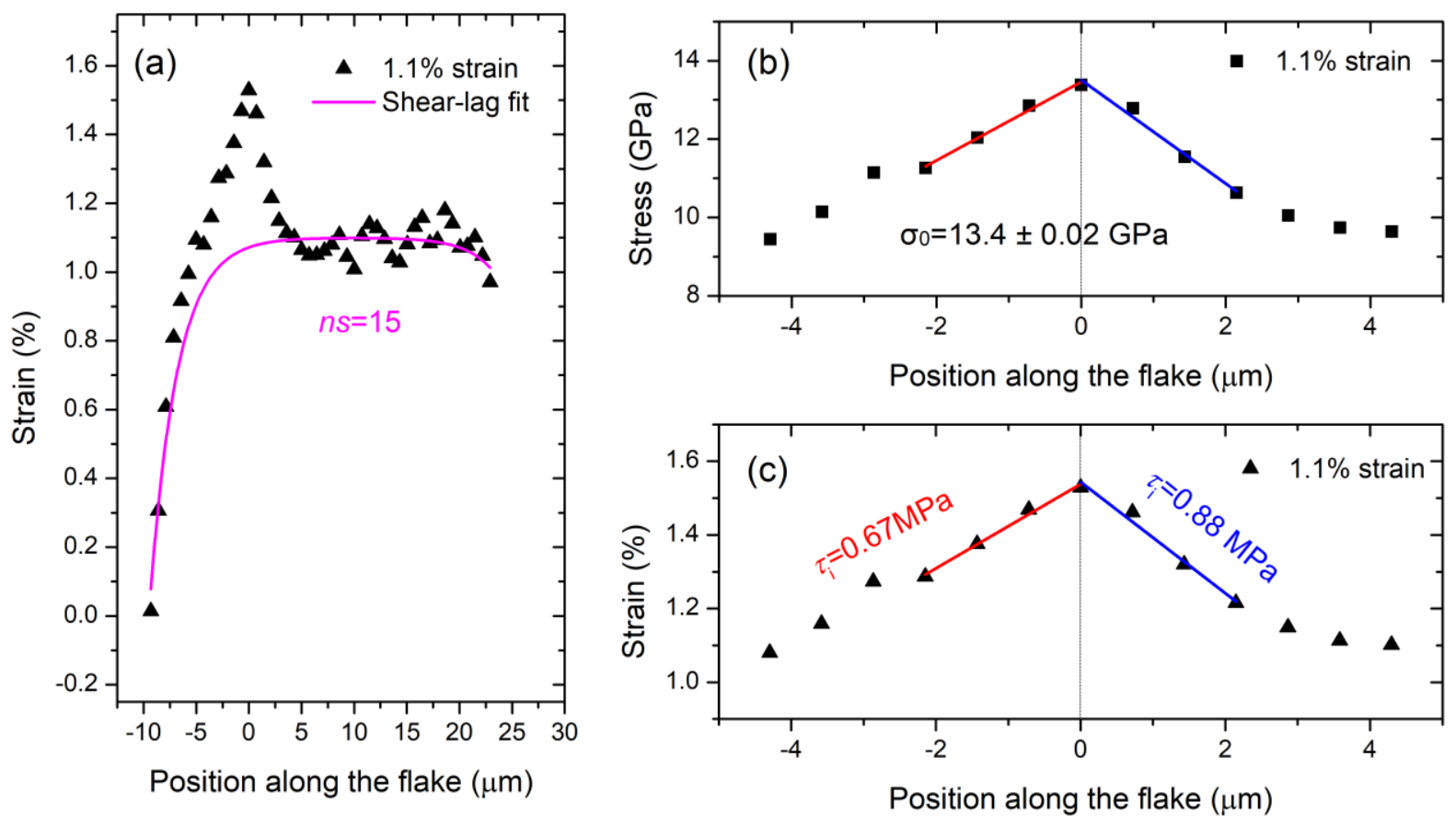

Figure S9. Analysis of flake No.5 (Table S1).
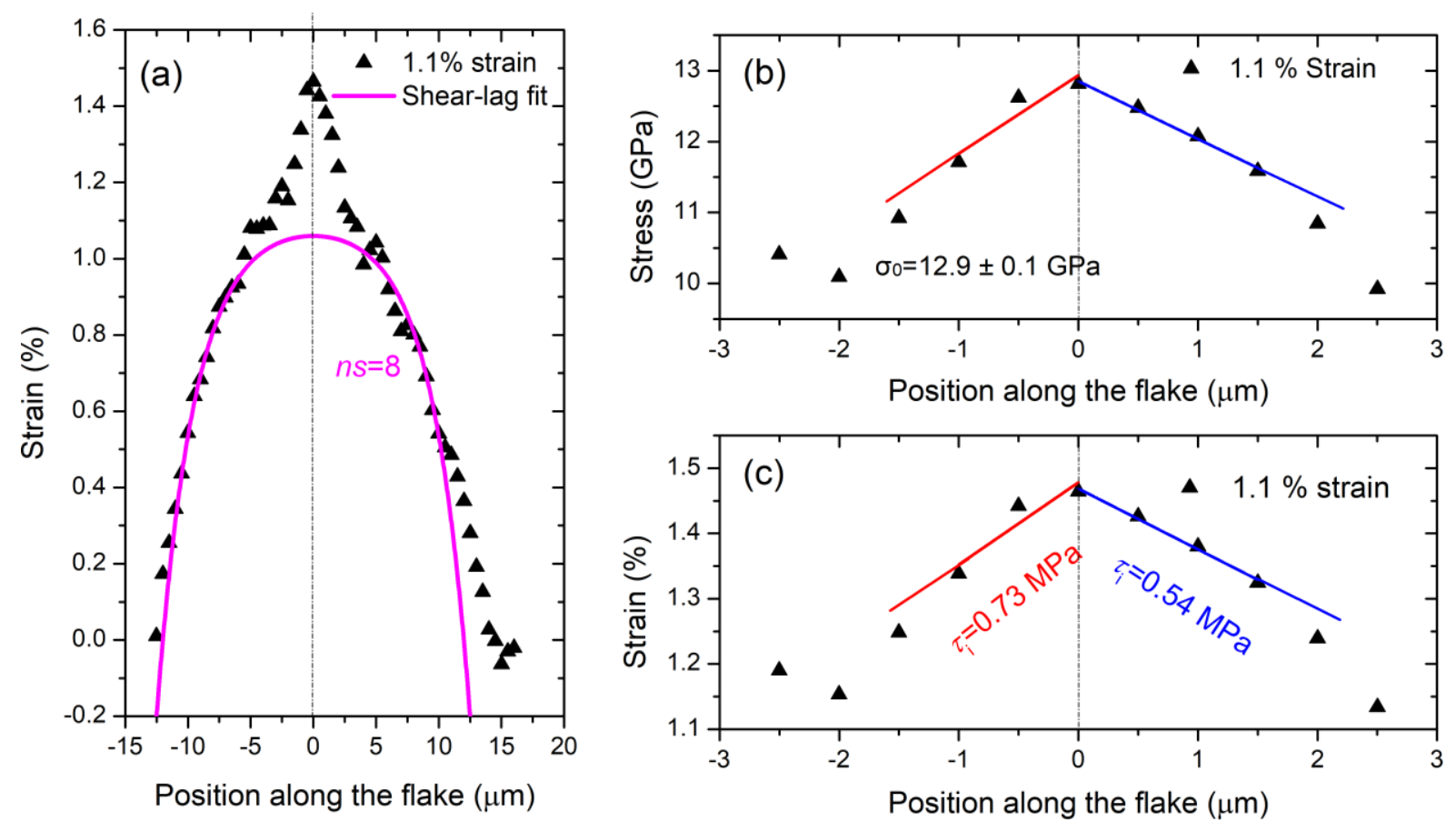

Figure S10. Analysis of flake No.6 (Table S1). 
It can be seen that the value of $G_{\mathrm{i}}$ reported in this work is generally in the order of $10^{-2} \mathrm{~J} / \mathrm{m}^{2}$, and $G_{\mathrm{i}}$ increases with $N$ as expected from earlier work, ${ }^{23}$ due to the extra contribution of the interlayer sliding (Figure S11). These values are comparable to those measured experimentally for graphene on a polymer substrate. ${ }^{24}$

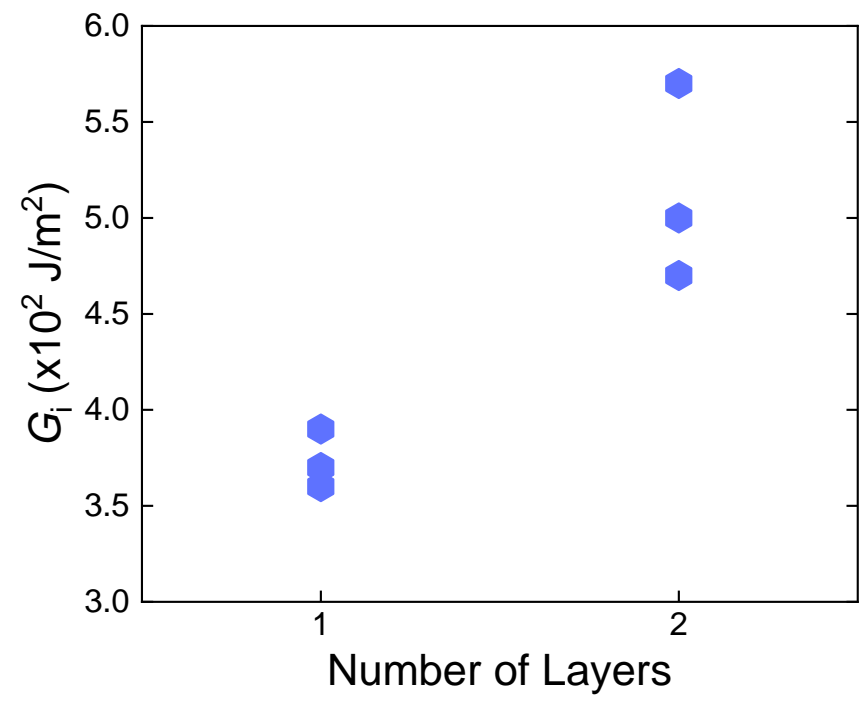

Figure $\mathrm{S} 11 G_{\mathrm{i}}$ as the function of $N$.

\section{S7. Applications of Strain Sensors}

In order to achieve practical fast scanning, line scans in addition to the areal mapping can also be employed. Similar to that shown in Figure 5 in the main text, strain sensors made using bilayer graphene (Figure S12a) have been used to successfully monitor the localized strain variation due to the formation of small crazes. It can be seen in Figure S12b that generally the strain keeps increasing and the debonding does not initiate until an applied strain of $\sim 1.2 \%$. Similarly, a strain sensor in the form of two long graphene strips monitored the strain increasing from $0 \%$ to $0.85 \%$ strain, and at $0.85 \%$ strain a craze initiates and crosses the two graphene strips (Figure S12c). It can be seen in Figure S12d that after keeping the specimen at $0.85 \%$ applied strain for 1.5 hours, the stress over the craze regions relaxes. ${ }^{25}$ 

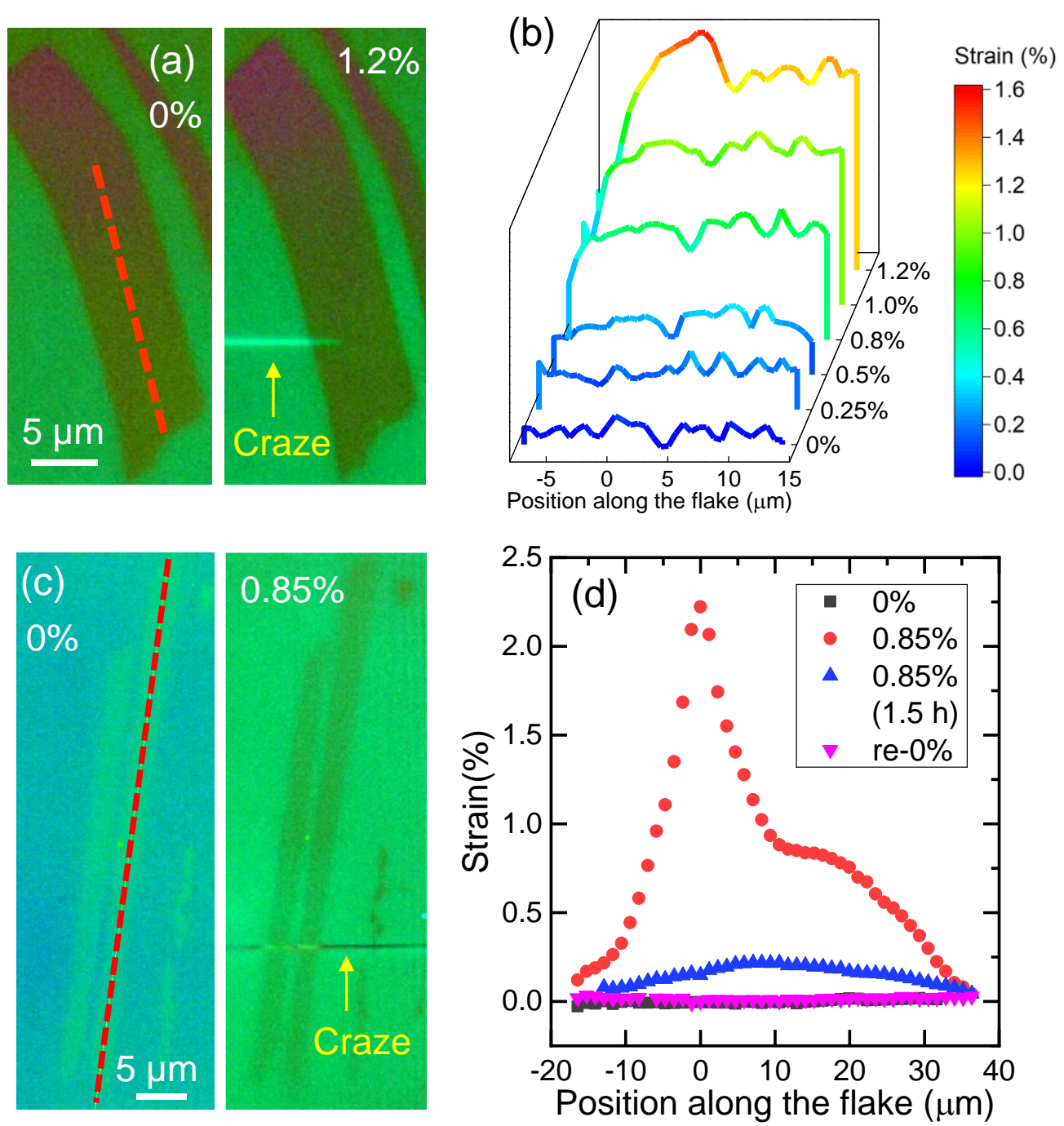

Figure S12 (a) False color optical micrograph of a bi-layer graphene at $0 \%$ and $1.2 \%$ applied strain, respectively. (b) Strain variations along the red line shown in (a) at different applied strains. (c) False color optical micrograph of ribbon-like monolayers at $0 \%$ and $0.85 \%$ applied strain, respectively. (d) Strain distribution along the red line shown in (c) at $0 \%$ and $0.85 \%$ applied strain, $0.85 \%$ applied strain after 1.5 hour, and released at $0 \%$ strain.

The applications of the mono- or few-layer graphene can also be compared with strain sensors based on graphene networks. ${ }^{26-31}$ They allow strain to be transferred from the target more efficiently and measured by Raman spectroscopy. This combination endows the excellent sensitivity, i.e. spectral and spatial resolution. These are the two advantages over the graphene network sensors, which have lower 'sensitivity' because (1) the weaker interfacial bonding between graphene and target does not transfer stress/strain well, hence small strains may not be detected; (2) the commonly used output signal - electrical conductivity measures larger area 
of graphene network sensors with poorer spatial resolution therefore it is not sufficiently sensitive to local strain variations caused by small defects such as those demonstrated in this work..$^{32}$

Strain sensors based on graphene networks use electrical measurement for an overall applied strain at global scale, hence they better perform at large scale to reduce the deviation or uniformity. It can, however, be difficult to measure localized strain variations from small defects as shown in this work. In contrast, the graphene sensors demonstrated in this work are particularly useful for small devices such as origami/kirigami structures and the similar device configurations, ${ }^{33-35}$ where precise measurement of localized strain is of great value. For the applications at that scale, the quality of graphene can be actually quite good and repeatable. Even devices with large dimension can also be acceptable as long as they fit on an optical microscope connected to Raman spectrometer. The advantages of using Raman spectroscopy are that it is non-destructive and the test can be carried out throughout the whole graphene flake to detect defects in the target, without any prior knowledge of their exact locations. For such local measurement, non-contact electrical measurement may not have the spatial resolution sufficiently sensitive to local strain variations caused by small defects such as those demonstrated in this work; ${ }^{32}$ or it requires the extra steps of depositing electrodes, which is practically difficult without prior knowledge of the exact location of small defects. Another side benefit for the method shown in this work is that in devices that have already employed graphene for other purposes, the existing graphene can be directly made used of to read out the strain distribution in combination with Raman spectroscopy.

The strain sensor based on mono- and few-layer graphene is a proof of concept using the simplest tape-exfoliated graphene with few defects to investigate the fundamental mechanism, but its realization is also feasible using other types of graphene. This is because the sensing relies on the measurement of the graphene strain using Raman spectroscopy along with the 
calibration between the strain and Raman 2D band position. For example, this band shift calibration might be, though not necessarily, affected by the presence of defects, vacancies, grain boundary, or different number of layers in CVD graphene, but once the new calibration is established as extensively investigated before, ${ }^{5,12,36,37}$ the realization of the strain sensors using CVD graphene is possible.

\section{S8. Strategies for Controlled Bandgap Opening}

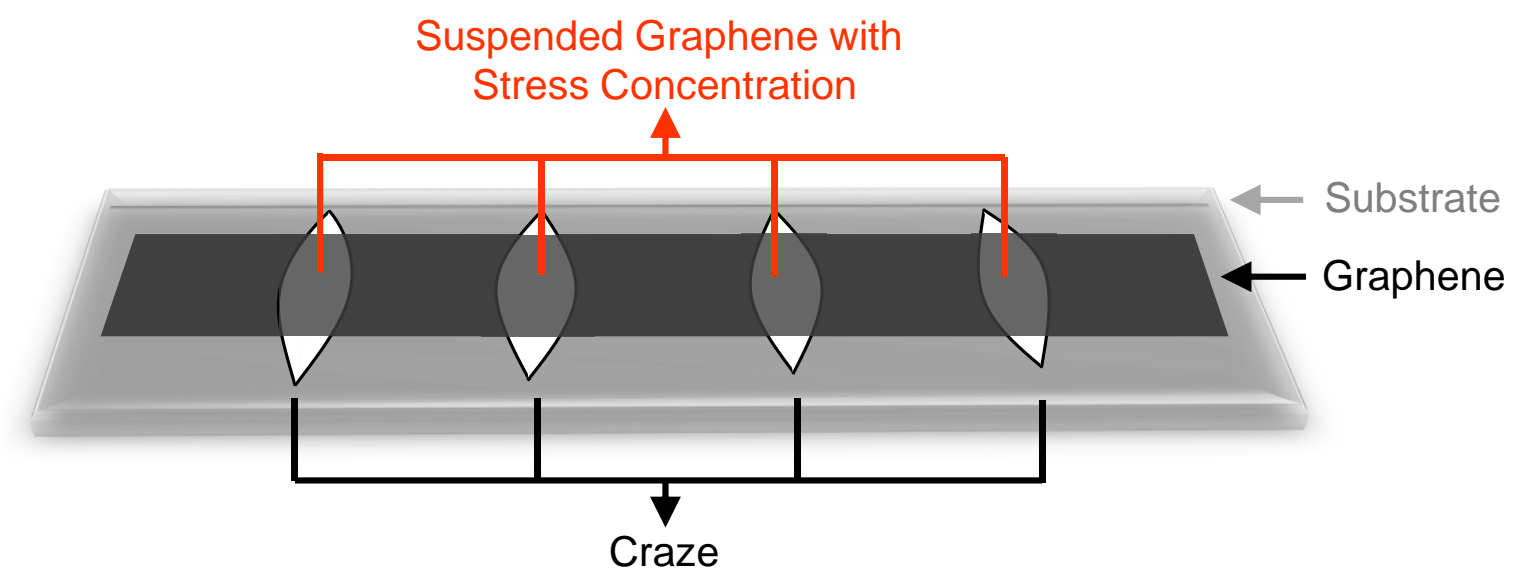

Figure S13 Schematic of the control of stress concentration and bandgap opening by engineering arrays of crazes in substrates.

\section{References}

1. Li, Z.; Young, R. J.; Papageorgiou, D. G.; Kinloch, I. A.; Zhao, X.; Yang, C.; Hao, S., Interfacial stress transfer in strain engineered wrinkled and folded graphene. 2D Materials 2019, 6 (4), 045026.

2. Kinloch, A. J.; Young, R. J., Fracture Behaviour of Polymers. Springer Science \& Business Media: 2013.

3. Gong, L.; Kinloch, I. A.; Young, R. J.; Riaz, I.; Jalil, R.; Novoselov, K. S., Interfacial Stress Transfer in a Graphene Monolayer Nanocomposite. Advanced Materials 2010, 22 (24), 2694-2697.

4. Mohiuddin, T. M. G.; Lombardo, A.; Nair, R. R.; Bonetti, A.; Savini, G.; Jalil, R.; Bonini, N.; Basko, D. M.; Galiotis, C.; Marzari, N.; Novoselov, K. S.; Geim, A. K.; Ferrari, A. C., Uniaxial strain in graphene by Raman spectroscopy: $G$ peak splitting, Grüneisen parameters, and sample orientation. Physical Review B 2009, 79 (20), 205433. 
5. Li, Z.; Kinloch, I. A.; Young, R. J.; Novoselov, K. S.; Anagnostopoulos, G.; Parthenios, J.; Galiotis, C.; Papagelis, K.; Lu, C.-Y.; Britnell, L., Deformation of Wrinkled Graphene. ACS Nano 2015, 9 (4), 3917-3925.

6. Ferrari, A. C.; Meyer, J. C.; Scardaci, V.; Casiraghi, C.; Lazzeri, M.; Mauri, F.; Piscanec, S.; Jiang, D.; Novoselov, K. S.; Roth, S.; Geim, A. K., Raman Spectrum of Graphene and Graphene Layers. Physical Review Letters 2006, 97 (18), 187401.

7. Yoon, D.; Son, Y.-W.; Cheong, H., Strain-Dependent Splitting of the DoubleResonance Raman Scattering Band in Graphene. Physical Review Letters 2011, 106 (15), 155502.

8. Young, R. J.; Liu, M.; Kinloch, I. A.; Li, S.; Zhao, X.; Vallés, C.; Papageorgiou, D. G., The mechanics of reinforcement of polymers by graphene nanoplatelets. Composites Science and Technology 2018, 154, 110-116.

9. Wang, L.; Zihlmann, S.; Baumgartner, A.; Overbeck, J.; Watanabe, K.; Taniguchi, T.; Makk, P.; Schönenberger, C., In Situ Strain Tuning in hBN-Encapsulated Graphene Electronic Devices. Nano Letters 2019, 19 (6), 4097-4102.

10. Zhang, Z.; Zhang, X.; Wang, Y.; Wang, Y.; Zhang, Y.; Xu, C.; Zou, Z.; Wu, Z.; Xia, Y.; Zhao, P.; Wang, H. T., Crack Propagation and Fracture Toughness of Graphene Probed by Raman Spectroscopy. ACS Nano 2019, 13 (9), 10327-10332.

11. Goldsche, M.; Sonntag, J.; Khodkov, T.; Verbiest, G. J.; Reichardt, S.; Neumann, C.; Ouaj, T.; von den Driesch, N.; Buca, D.; Stampfer, C., Tailoring Mechanically Tunable Strain Fields in Graphene. Nano Letters 2018, 18 (3), 1707-1713.

12. Neumann, C.; Reichardt, S.; Venezuela, P.; Drögeler, M.; Banszerus, L.; Schmitz, M.; Watanabe, K.; Taniguchi, T.; Mauri, F.; Beschoten, B.; Rotkin, S. V.; Stampfer, C., Raman spectroscopy as probe of nanometre-scale strain variations in graphene. Nature Communications 2015, 6 (1), 8429.

13. González-Chi, P. I.; Young, R. J., Crack bridging and fibre pull-out in polyethylene fibre reinforced epoxy resins. Journal of Materials Science 1998, 33 (24), 5715-5729.

14. Hsueh, C.-H., Crack-wake interfacial debonding criteria for fiber-reinforced ceramic composites. Acta Materialia 1996, 44 (6), 2211-2216.

15. Lee, J. E.; Ahn, G.; Shim, J.; Lee, Y. S.; Ryu, S., Optical separation of mechanical strain from charge doping in graphene. Nature Communications 2012, 3 (1), 1024.

16. Wang, Y.; Wang, Y.; Xu, C.; Zhang, X.; Mei, L.; Wang, M.; Xia, Y.; Zhao, P.; Wang, H., Domain-boundary independency of Raman spectra for strained graphene at strong interfaces. Carbon 2018, 134, 37-42.

17. Si, C.; Sun, Z.; Liu, F., Strain engineering of graphene: a review. Nanoscale 2016, 8 (6), 3207-3217.

18. Li, Y.; He, T.; Shi, L.; Wang, R.; Sun, J., Strain Sensor with Both a Wide Sensing Range and High Sensitivity Based on Braided Graphene Belts. ACS Applied Materials \& Interfaces 2020, 12 (15), 17691-17698.

19. Wang, F.; Li, S.; Bissett, M. A.; Kinloch, I. A.; Li, Z.; Young, R. J., Strain engineering in monolayer WS2 and WS2 nanocomposites. 2D Materials 2020, 7 (4), 045022.

20. Gong, L.; Young, R. J.; Kinloch, I. A.; Riaz, I.; Jalil, R.; Novoselov, K. S., Optimizing the Reinforcement of Polymer-Based Nanocomposites by Graphene. ACS Nano 2012, 6 (3), 2086-2095.

21. Neek-Amal, M.; Peeters, F. M., Nanoindentation of a circular sheet of bilayer graphene. Physical Review B 2010, 81 (23), 235421.

22. Androulidakis, C.; Koukaras, E. N.; Rahova, J.; Sampathkumar, K.; Parthenios, J.; Papagelis, K.; Frank, O.; Galiotis, C., Wrinkled Few-Layer Graphene as Highly Efficient Load Bearer. ACS Applied Materials \& Interfaces 2017, 9 (31), 26593-26601. 
23. Li, Y.; Huang, S.; Wei, C.; Wu, C.; Mochalin, V. N., Adhesion of two-dimensional titanium carbides (MXenes) and graphene to silicon. Nature Communications 2019, 10 (1), 3014.

24. Jiang, T.; Huang, R.; Zhu, Y., Interfacial Sliding and Buckling of Monolayer Graphene on a Stretchable Substrate. Advanced Functional Materials 2014, 24 (3), 396-402.

25. Donald, A. M.; Kramer, E. J., Effect of strain history on craze microstructure. Polymer 1982, 23 (3), 457-460.

26. Boland, C. S.; Khan, U.; Ryan, G.; Barwich, S.; Charifou, R.; Harvey, A.; Backes, C.; Li, Z.; Ferreira, M. S.; Möbius, M. E.; Young, R. J.; Coleman, J. N., Sensitive electromechanical sensors using viscoelastic graphene-polymer nanocomposites. Science 2016, 354 (6317), 1257.

27. Wang, M. C.; Chun, S.; Han, R. S.; Ashraf, A.; Kang, P.; Nam, S., Heterogeneous, Three-Dimensional Texturing of Graphene. Nano Letters 2015, 15 (3), 1829-1835.

28. Wang, Y.; Wang, L.; Yang, T.; Li, X.; Zang, X.; Zhu, M.; Wang, K.; Wu, D.; Zhu, H., Wearable and Highly Sensitive Graphene Strain Sensors for Human Motion Monitoring. Advanced Functional Materials 2014, 24 (29), 4666-4670.

29. Biccai, S.; Boland, C. S.; O’Driscoll, D. P.; Harvey, A.; Gabbett, C.; O’Suilleabhain, D. R.; Griffin, A. J.; Li, Z.; Young, R. J.; Coleman, J. N., Negative Gauge Factor Piezoresistive Composites Based on Polymers Filled with MoS2 Nanosheets. ACS Nano 2019, 13 (6), 6845-6855.

30. Hempel, M.; Nezich, D.; Kong, J.; Hofmann, M., A Novel Class of Strain Gauges Based on Layered Percolative Films of 2D Materials. Nano Letters 2012, 12 (11), 5714-5718. 31. O’Mara, M. A.; Ogilvie, S. P.; Large, M. J.; Amorim Graf, A.; Sehnal, A. C.; Lynch, P. J.; Salvage, J. P.; Jurewicz, I.; King, A. A. K.; Dalton, A. B., Ultrasensitive Strain Gauges Enabled by Graphene-Stabilized Silicone Emulsions. Advanced Functional Materials 2020, 30 (32), 2002433.

32. Melios, C.; Huang, N.; Callegaro, L.; Centeno, A.; Cultrera, A.; Cordon, A.; Panchal, V.; Arnedo, I.; Redo-Sanchez, A.; Etayo, D.; Fernandez, M.; Lopez, A.; Rozhko, S.; Txoperena, O.; Zurutuza, A.; Kazakova, O., Towards standardisation of contact and contactless electrical measurements of CVD graphene at the macro-, micro- and nano-scale. Scientific Reports 2020, 10 (1), 3223.

33. Blees, M. K.; Barnard, A. W.; Rose, P. A.; Roberts, S. P.; McGill, K. L.; Huang, P. Y.; Ruyack, A. R.; Kevek, J. W.; Kobrin, B.; Muller, D. A.; McEuen, P. L., Graphene kirigami. Nature 2015, 524 (7564), 204-207.

34. Kang, D.; Pikhitsa, P. V.; Choi, Y. W.; Lee, C.; Shin, S. S.; Piao, L.; Park, B.; Suh, K.-Y.; Kim, T.-i.; Choi, M., Ultrasensitive mechanical crack-based sensor inspired by the spider sensory system. Nature 2014, 516 (7530), 222-226.

35. Fan, X.; Forsberg, F.; Smith, A. D.; Schröder, S.; Wagner, S.; Rödjegård, H.; Fischer, A. C.; Östling, M.; Lemme, M. C.; Niklaus, F., Graphene ribbons with suspended masses as transducers in ultra-small nanoelectromechanical accelerometers. Nature Electronics 2019, 2 (9), 394-404.

36. Bronsgeest, M. S.; Bendiab, N.; Mathur, S.; Kimouche, A.; Johnson, H. T.; Coraux, J.; Pochet, P., Strain Relaxation in CVD Graphene: Wrinkling with Shear Lag. Nano Letters 2015, 15 (8), 5098-5104.

37. Androulidakis, C.; Koukaras, E. N.; Paterakis, G.; Trakakis, G.; Galiotis, C., Tunable macroscale structural superlubricity in two-layer graphene via strain engineering. Nature Communications 2020, 11 (1), 1595. 